\title{
$A$-TRANSFORMS AND HILBERT FUNCTIONS IN LOCAL LATTICES
}

\author{
BY \\ E. W. JOHNSON
}

0. Introduction. In [1], Dilworth introduced the notion of a principal element of a multiplicative lattice and used it to define a class of lattices in which the Noether decomposition theorems hold. He called these Noether lattices and showed, among other things, that Noether lattices satisfy the Krull Intersection Theorem and the Krull Principal Ideal Theorem.

Noether lattices in general, however, are lacking in many important ring-like properties. For example, in a local Noether lattice of altitude $d$, there need not exist $d$ principal elements with join an element which is primary for the maximal element, so that systems of parameters may be much larger than one would hope.

In this paper it is shown that if $P$ is the maximal element of a local Noether lattice, then for large $n$ the number of principal elements required to generate $P^{n}$ is given by a polynomial $B^{*}(P, x)$ (Corollary 3.3). It seems reasonable then to consider the degree of this polynomial as a second measure of the number of elements in a system of parameters, or possibly as an alternative definition of the dimension of the lattice. Lemma 3.6 and Theorem 3.9 establish that the degree of $B^{*}(P, x)$ is $d-1$ where $d$ is the height of $P$.

$\S 2$ is concerned with the introduction of a graded multiplicative lattice, denoted $\mathscr{R}(\mathscr{L}, A)$. This lattice is analogous to the $A$-transform of a Noetherian ring introduced by $D$. Rees and serves as the vehicle in $\$ 3$ for obtaining the polynomial $B^{*}(P, x)$. The main result of this section is that, if $\mathscr{L}$ is a Noether lattice, then $\mathscr{R}(\mathscr{L}, A)$ is a Noether lattice (Theorem 2.11). A determination is also made of the heights of the maximal primes of $\mathscr{R}(\mathscr{L}, A)$ in terms of $A$ and $\mathscr{L}$ (Lemma 2.16).

In $\$ 4$ regular local lattices are considered. It is shown that the 0 -element of a regular local lattice is prime (Theorem 4.1) and the results of $\$ 2$ are applied to show that the degree function is a valuation (Corollary 4.4).

The author expresses his gratitude to Professor Louis J. Ratliff for many helpful suggestions.

Throughout the remainder of this paper, the symbol $\mathscr{L}$ will denote a Noether lattice.

1. The following lemma is a useful generalization of Lemma (6.3) in [1].

Lemma 1.1. Let $A, B$ and $C$ be elements of $\mathscr{L}$ with $A$ principal, $B \leqq C$ and $(0: A)$ $\wedge C \leqq B$. Then the correspondence $D \rightarrow A D$ of the quotient $C / B$ into the quotient $C A \mid B A$ is a lattice isomorphism.

Received by the editors February 8, 1967 and, in revised form, December 8, 1967. 
Proof. Clearly the correspondence is order preserving. Let $D$ and $E$ be elements of $C / B$. Then since $A$ is principal and $(0: A) \wedge C \leqq B, D=D \vee((0: A) \wedge C)$ $=C \wedge(D \vee(0: A))=C \wedge(D A: A)$, and similarly, $E=C \wedge(E A: A)$. Hence if $D A \leqq E A$, then $D \leqq E$, and if $D A=E A$, then $D=E$. Now, let $F$ be any element of $C A / B A$. Then $(F: A) \wedge C$ is an element of $C / B$ and $((F: A) \wedge C) A=F \wedge C A=F$, since $A$ is principal. Hence $C / B \cong C A / B A$. Q.E.D.

If $B, C$ are elements of $\mathscr{L}$ with $B<C$, then $C$ will be called a cover for $B$ if $B \leqq X<C$ implies $B=X$. The following theorem characterizes the covering relation in $\mathscr{L}$.

TheOREM 1.2. Let $B$ and $C$ be elements of $\mathscr{L}$ with $B<C$. Then $C$ is a cover for $B$ if, and only if, there exists a principal element $A \in \mathscr{L}$ with $B \vee A=C$ such that $B: A$ is maximal in $\mathscr{L}$.

Proof. If $A$ is principal in $\mathscr{L}$, then by Lemma $1.1,(B \vee A) / B \cong A I /(B \wedge A)=$ $A I /(B: A) A \cong I /(B: A)$. The theorem follows immediately from this. Q.E.D.

In [1] it was shown that Noether lattices satisfy an abstract "Krull Intersection Theorem." An immediate but useful consequence is stated for ease of reference in the following

Lemma 1.3. If $\mathscr{L}$ is a local lattice and if $B, C$, and $D$ are elements of $\mathscr{L}$ with $D<I$, then $B \leqq C \vee B D$ implies $B \leqq C$.

Proof. $B \leqq C \vee B D$ implies $B \leqq C \vee B D^{n}$ for all $n$, so that necessarily $B \leqq C$. Q.E.D.

If an element $B \in \mathscr{L}$ is the join of principal elements $A_{1}, \ldots, A_{K}$ then the elements $A_{i}$ will be called a base or basis for $B$. If this is the case and if no proper subcollection of the $A_{i}$ also form a basis for $B$, then the $A_{i}$ will be said to be a minimal base for $B$. As for rings, it is clear that any base can be reduced to a minimal base.

TheOREM 1.4. Let $\mathscr{L}$ be a local lattice with maximal element $P$ and let $A \leqq P$ be an element of $\mathscr{L}$. Then

1. the quotient $A / A P$ is finite dimensional and

2. the number of elements in any minimal base for $A$ is equal to the dimension of $A \mid A P$.

Proof. Let $A_{1}, \ldots, A_{r}$ be a minimal base for $A$. Set $S_{0}=A P$ and for $1 \leqq i \leqq r$, let $S_{i}=S_{i-1} \vee A_{i}$. Then $P A_{i} \leqq P A \leqq S_{i-1}$, and hence $S_{i-1}: A_{i} \geqq P(1 \leqq i \leqq r)$. Since $P$ is maximal, it follows that either $S_{i-1}: A_{i}=P$ or $S_{i-1}: A_{i}=I$ and hence that either $S_{i}=S_{i-1} \vee A_{i}$ is a cover for $S_{i-1}$ (Theorem 1.2) or that $S_{i}=S_{i-1}$. It is immediate then that $A P=S_{0} \leqq S_{1} \leqq \cdots \leqq S_{r}=A$ can be reduced to a composition chain from $A P$ to $A$ and hence that the quotient $A / A P$ is finite dimensional with dimension (say) $d \leqq r$. 
Assume now that $d<r$. Then for some $i, 1 \leqq i \leqq r, S_{i}=S_{i-1}$. Hence

$$
\begin{aligned}
A & =S_{i} \vee A_{i+1} \vee \cdots \vee A_{r} \\
& =S_{i+1} \vee A_{i+1} \vee \cdots \vee A_{r} \\
& =A P \vee A_{1} \vee \cdots \vee A_{i-1} \vee A_{i+1} \vee \cdots \vee A_{r} .
\end{aligned}
$$

But then by Lemma $1.3, A=A_{1} \vee \cdots \vee A_{i-1} \vee A_{i+1} \vee \cdots \vee A_{r}$, which contradicts the minimality of the base $A_{1}, \ldots A_{r}$. It follows, therefore, that the number of elements in any minimal base for $A$ is the dimension of the quotient $A / A P$. Q.E.D.

Corollary 1.5. Let $\mathscr{L}$ be a local lattice and let $\mathscr{C}$ be a collection of principal elements with the property that for every element $A \in \mathscr{L}$ there exist elements $A_{1}, \ldots, A_{k}$ (depending on $A$ ) in $\mathscr{C}$ such that $A=A_{1} \vee \cdots \vee A_{k}$. Then $\mathscr{C}$ is the complete collection of principal elements of $\mathscr{L}$.

Proof. If $B$ and $A_{1}, \ldots, A_{k}$ are principal elements of $\mathscr{L}$ with $B=A_{1} \vee \cdots \vee A_{k}$, then $A_{1}, \ldots, A_{k}$ can be reduced to a minimal base for $B$. Since $B$ is itself principal, it follows that $B=A_{i}$, for some $i$. Q.E.D.

COROllary 1.6. Let $R$ be a local ring, and let $\mathscr{L}$ be the lattice of the ideals of $R$. Then the principal elements of $\mathscr{L}$ are the principal ideals of $R$.

Proof. It was established in [1] that the principal ideals of $R$ are principal elements of $\mathscr{L}$, and it follows from Corollary 1.5 that these are the only principal elements. Q.E.D.

If $B \in \mathscr{L}$, then as shown in [1], the quotient $I / B$ becomes a Noether lattice, denoted $\mathscr{L} \mid B$, if multiplication is defined by $C \circ D=C D \vee B$. The principal elements of $\mathscr{L} \mid B$ include the elements $A \vee B$ where $A$ is principal in $\mathscr{L}$. Since some confusion will naturally result about the multiplication when more than one quotient lattice $\mathscr{L} \mid B$ is under consideration at the same time, the notation $C / B$ will be used to denote the element $C \vee B$ of $\mathscr{L} \mid B$ when it is necessary to distinguish. With this notation, if $A$ is principal in $\mathscr{L}$, then $A / B$ is principal in $\mathscr{L} / B$.

CoROllary 1.7. If an element $B \in \mathscr{L}$ is such that $\mathscr{L} \mid B$ is local, then the principal elements of $\mathscr{L} \mid B$ are the elements $A \mid B$ with $A$ principal in $\mathscr{L}$.

Proof. The corollary is immediate from Corollary 1.5. Q.E.D.

If an element $P$ of $\mathscr{L}$ is prime (i.e., $A B \leqq P$ implies $A \leqq P$ or $B \leqq P$ ), then the height of $P$, denoted ht $P$, is defined as the supremum of all integers $n$ for which there exists a prime chain $P_{0}<P_{1}<\cdots<P_{n}=P$ in $\mathscr{L}$.

Lemma 1.8. Let $P$ be a prime element of $\mathscr{L}$ and let $A$ be a principal element with $A \leqq P$. Then

$$
\text { ht } P \geqq \text { ht } P / A \geqq \text { ht } P-1 \text {. }
$$

If $A \leqq P^{\prime}$ for every minimal associated prime $P^{\prime} \leqq P$ of 0 , then ht $P / A=$ ht $P-1$. 
Proof. It was shown in [1] that an element $C / A$ is prime or primary in $\mathscr{L} \mid A$ if, and only if, the element $C \vee A$ is respectively prime or primary in $\mathscr{L}$. In particular then, the element $P=P / A$ is prime in $\mathscr{L} \mid A$. Now, let $d=$ ht $P$ and let $P_{0}<P_{1}<\cdots$ $<P_{d}=P$ be a prime chain in $\mathscr{L}$. Then (by Lemma 6.4 of [1]), there exists a prime chain $P_{0}^{*}<P_{1}^{*}<\cdots<P_{d}^{*}=P$ in $\mathscr{L}$ with $P_{0}=P_{0}^{*}$ and $A \leqq P_{1}^{*}$. It is immediate from this that ht $P / A \geqq d-1$. Since ht $P / A \leqq$ ht $P$ is clear, the first statement is proved. Now, if ht $P / A=d$, then there is a prime sequence $P_{0}^{\prime}<P_{1}^{\prime}<\cdots<P_{d}^{\prime}=P$ in $\mathscr{L} / A$ and therefore also in $\mathscr{L}$, with $A \leqq P_{0}^{\prime}$. Since $P_{0}^{\prime}$ is necessarily a minimal associated prime of 0 in $\mathscr{L}$, the second statement follows. Q.E.D.

2. Let $\mathscr{L}$ denote a Noether lattice with least element 0 and greatest element $I$, and let $A$ be an arbitrary element of $\mathscr{L}$. In this section a lattice analogue of D. Rees' $A$-transform of a Noetherian ring $R$ by an ideal $A$ [4] will be introduced. It is this transform, denoted $\mathscr{R}(\mathscr{L}, A)$, which will form the basis for the later proof of the existence of Hilbert Characteristic Polynomials.

Let $\mathscr{F}(A)$ denote the collection of all formal sums $\sum_{i=-\infty}^{\infty} B_{i}$ of elements of $\mathscr{L}$ such that

(2.1) $A^{i} \geqq B_{i} \geqq B_{i+1} \geqq A B_{i}$, for all $i$, where for $i \leqq 0, A^{i}=I$.

The elements of $\mathscr{F}(A)$ will generally be denoted by capitals $B, C, \ldots$, and if $B \in \mathscr{F}(A)$, then $B_{i}$ will denote the $i$ th coordinate of $B$. For elements $B, C \in \mathscr{F}(A)$ the following definitions will be used:

(2.2) $B \leqq C$ if $B_{i} \leqq C_{i}$ for all $i$,

(2.3) $B \vee C=\sum\left(B_{i} \vee C_{i}\right)$,

(2.4) $B \wedge C=\sum\left(B_{1} \wedge C_{i}\right)$,

(2.5) $B \cdot C=\sum_{i}\left(\bigvee_{r+s=i} B_{r} C_{s}\right)$.

It is seen that the collection $\mathscr{F}(A)$ forms a complete, modular, lattice under the relation $\leqq$ with resulting join and meet given by (2.3) and (2.4), and that (2.5) defines a commutative, associative multiplication on $\mathscr{F}(A)$ which distributes over the join operation (2.3). The resulting lattice, together with the multiplication (2.5) will be denoted by the symbol $\mathscr{R}(\mathscr{L}, A)$ and will be called the $A$-transform of $\mathscr{L}$, It is clear then from the defining relations that $\mathscr{R}(\mathscr{L}, A)$ has greatest element $I^{*}=\sum A^{i}$. The element $I^{*}$ is also a multiplicative identity, since by $(2.1)$ and (2.5) it is seen that if $B$ is any element of $\mathscr{R}(\mathscr{L}, A)$, then the $i$ th coordinate of $B I^{*}$ is $\bigvee_{r+s=i} B_{r} I_{s}^{*}=\bigvee_{r+s=i} B_{r} A^{s}=B_{i}$. These comments are summarized in the following

LEMMA 2.1. $\mathscr{R}(\mathscr{L}, A)$ is a complete, modular, multiplicative lattice.

Definition 2.2. Let $C$ be an element of $\mathscr{L}$ with $C \leqq A^{r}$. Then $C^{(r)}$ is the least element $D$ of $\mathscr{R}(\mathscr{L}, A)$ such that $C \leqq D_{r}$. The notation $C_{A}^{(r)}$ will also be used when it is necessary to indicate that $C^{(r)}$ is to be computed in $\mathscr{R}(\mathscr{L}, A)$.

The following lemmas will be useful in computations.

Lemma 2.3. If $C \leqq A^{r}$ in $\mathscr{L}$, then $C^{(r)}=\sum C A^{i-r}$. If also $D \leqq A^{r}$, then $C^{(r)} \vee D^{(r)}$ $=(C \vee D)^{(r)}$. 
Proof. Clearly $\sum C A^{i-r} \in \mathscr{R}(\mathscr{L}, A)$, so in view of (2.1) it follows that $C^{(r)}=\sum C A^{i-r}$. If also $D \leqq A^{r}$, so that $D^{(r)}=\sum D A^{i-r}$, then by (2.3)

$$
C^{(r)} \vee D^{(r)}=\sum(C \vee D) A^{i-r}=(C \vee D)^{(r)} \quad \text { Q.E.D. }
$$

Lemma 2.4. If $B \in \mathscr{R}(\mathscr{L}, A)$ and if $C \in \mathscr{L}$ is such that $C \leqq A^{r}$, then $B C^{(r)}=\sum B_{i-r} C$.

Proof. $B C^{(r)}$ has $i$ th coordinate $\bigvee_{s+t=i} B_{s} C^{(r)}{ }_{t}$. Let $s+t=i$. If $s>i-r$ so that $t<r$, then $B_{s} C^{(r)}{ }_{t}=B_{s} C \leqq B_{i-r} C$ by (2.1). On the other hand, if $s<i-r$ so that $t>r$, then $B_{s} C^{(r)}{ }_{t}=B_{s} C A^{t-r} \leqq B_{s+t-r} C=B_{i-r} C$. If $s=i-r$ so that $t=r$, then $B_{s} C^{(r)}{ }_{t}$ $=B_{i-r} C$. Hence

$$
B_{i-r} C \leqq \bigvee_{s+t=i} B_{s} C^{(r)}{ }_{t} \leqq B_{i-r} C . \quad \text { Q.E.D. }
$$

CorollaRY 2.5. If $C \leqq A^{r}$ and $D \leqq A^{s}$ in $\mathscr{L}$, then $C^{(r)} D^{(s)}=(C D)^{(r+s)}$.

Proof. By Lemmas 2.3 and $2.4, C^{(r)} D^{(s)}$ has $i$ th coordinate $\left(C^{(r)}\right)_{i-s} D=C A^{i-s-r} D$ $=C D A^{i-(s+r)}$, which is the $i$ th coordinate of $(C D)^{(s+r)}$. Q.E.D.

Lemma 2.6. If $C \in \mathscr{R}(\mathscr{L}, A)$ and if $B \leqq A^{r}$ in $\mathscr{L}$, then $C: B^{(r)}=\sum_{i}\left[\left(C_{i+r}: B\right) \wedge A^{i}\right]$.

Proof. Let $D$ be any element of $\mathscr{R}(\mathscr{L}, A)$ such that $D B^{(r)} \leqq C$. Then $D_{i-r} B \leqq C_{i}$, for all $i$; and hence $D_{i-r} \leqq\left(C_{i}: B\right) \wedge A^{i-r}$, for all $i$. Now, set $E_{i}=\left(C_{i+r}: B\right) \wedge A^{i}$, for all $i$. Then $A^{i} \geqq E_{i} \geqq E_{i+1} \geqq A E_{i}$, for all $i$, so that $E=\sum E_{i} \in \mathscr{R}(\mathscr{L}, A)$. Since $\left(E B^{(r)}\right)_{i}$ $=E_{i-r} B=\left(\left(C_{i}: B\right) \wedge A^{i-r}\right) B \leqq C_{i}$, for all $i$, it now follows that $E=C: B^{(r)}$. Q.E.D.

THEOREM 2.7. Let $B$ be a principal element of $\mathscr{L}$ such that $B \leqq A^{r}$. Then $B^{(r)}$ is a principal element of $\mathscr{R}(\mathscr{L}, A)$.

Proof. Let $B \leqq A^{r}$ be a principal element of $\mathscr{L}$ and let $C$ and $D$ be any elements of $\mathscr{R}(\mathscr{L}, A)$. Then by Lemmas 2.4 and 2.6 ,

$$
\begin{aligned}
\left(\left(C \wedge\left(D: B^{(r)}\right)\right) B^{(r)}\right)_{i} & =\left(C \wedge\left(D: B^{(r)}\right)\right)_{i-r} B \\
& =\left(C_{i-r} \wedge\left(D_{i}: B\right) \wedge A^{i-r}\right) B=\left(C_{i-r} \wedge\left(D_{i}: B\right)\right) B \\
& =C_{i-r} B \wedge D_{i}=\left(C B^{(r)} \wedge D\right)_{i},
\end{aligned}
$$

for all $i$. Similarly,

$$
\begin{aligned}
\left(\left(C \vee D B^{(r)}\right): B^{(r)}\right)_{i} & =\left(\left(C_{i+r} \wedge D_{i} B\right): B\right) \wedge A^{i}=\left(\left(C_{i+r}: B\right) \vee D_{i}\right) \wedge A^{i} \\
& =\left(\left(C_{i+r}: B\right) \wedge A^{i}\right) \vee D_{i}=\left(\left(C: B^{(r)}\right) \vee D\right)_{i},
\end{aligned}
$$

for all $i$. Hence $B^{(r)}$ is principal. Q.E.D.

The following theorem will be instrumental in establishing that $\mathscr{R}(\mathscr{L}, A)$ satisfies the ascending chain condition.

THEOREM 2.8. Let $B$ be any element of $\mathscr{R}(\mathscr{L}, A)$. Then there exists an integer $N$ such that $B_{N+i}=B_{N} A^{i}$, for all $i \geqq 0$.

Proof. Let $\mathscr{F}$ be the family of all elements $B$ in $\mathscr{R}(\mathscr{L}, A)$ for which there fails to exist an integer $N$ such that $B_{N+i}=B_{N} A^{i}$, for all $i \geqq 0$. Assume $\mathscr{F} \neq \varnothing$. Let $C$ be any 
chain of elements of $\mathscr{F}$. Then it is easily seen that $S=\bigvee C \in \mathscr{F}$, for if not, then since $\mathscr{L}$ is Noetherian, there exist integers $N$ and $K$ such that $S_{i}=S_{K}$ for $i \leqq K$ and $S_{i}$ $=S_{N} A^{i-N}$, for $i \geqq N$, and for each $i, K \leqq i \leqq N$, there exists an element $C(i) \in C$ such that $C(i)_{i}=S_{i}$. Let $M$ be the maximum of the elements $C(i)$. Then $M \in C$ and $M_{i}$ $=S_{i}$ for $K \leqq i \leqq N$. Furthermore, if $i>N$, then $S_{i} \geqq M_{i} \geqq M_{N} A^{i-N}=S_{N} A^{i-N}=S_{i}$; and if $i<K$, then $S_{i} \geqq M_{i} \geqq M_{k}=S_{k}=S_{i}$. Hence $S_{i}=M_{i}$, for all $i$, so that $S=M \in \mathscr{F}$, which is a contradiction.

Hence if $\mathscr{F} \neq \varnothing$, then there exists a maximal element $F$ of $\mathscr{F}$. Then $F_{1}<A$ since $F_{1}=A$ implies $A^{n} \geqq F_{n} \geqq F_{1} A^{n-1}=A^{n}$, for all $n \geqq 1$, which contradicts $F \in \mathscr{F}$. Let $E$ be a principal element of $\mathscr{L}$ such that $F_{1}<F_{1} \vee E \leqq A$. Then $F<F \vee E^{(1)}$, so $F \vee E^{(1)}$ $\notin \mathscr{F}$ and there exists an integer $N$ such that $\left(F \vee E^{(1)}\right)_{n} A=\left(F \vee E^{(1)}\right)_{n+1}$, for all $n \geqq N$. Hence $\left(F_{n} \vee E A^{n-1}\right) A=F_{n} A \vee E A^{n}=F_{n+1} \vee E A^{n}$; as therefore $F_{n+1} \leqq$ $F_{n} A \vee E A^{n}$, for $n \geqq N$. Then since $\mathscr{L}$ is modular and $E$ is principal in $\mathscr{L}$,

$$
\begin{aligned}
F_{n+1}=F_{n+1} \wedge\left(F_{n} A \vee E A^{n}\right) & =F_{n} A \vee\left(F_{n+1} \wedge E A^{n}\right) \\
& =F_{n} A \vee\left(A^{n} \wedge\left(F_{n+1}: E\right)\right) E,
\end{aligned}
$$

for $n \geqq N$. Now, by Lemma $2.6, A^{n} \wedge\left(F_{n+1}: E\right)$ is the $n$th coordinate of the element $F: E^{(1)}$ of $\mathscr{R}(\mathscr{L}, A)$; so if $F: E^{(1)}=F$, then $F_{n}=A^{n} \wedge\left(F_{n+1}: E\right)$, for all $n$, so that by (2.6), $F_{n+1}=F_{n} A \vee F_{n} E=F_{n} A$, for all $n \geqq N$, in contradiction to the assumption that $F \in \mathscr{F}$. Hence $F: E^{(1)}>F$, so $F: E^{(1)} \notin \mathscr{F}$, and there exists an integer $N^{\prime} \geqq N$ such that

$$
A^{n} \wedge\left(F_{n+1}: E\right)=\left(A^{n-1} \wedge\left(F_{n}: E\right)\right) A, \text { for all } n \geqq N^{\prime} .
$$

But then by (2.6) and (2.7), for $n>N^{\prime}, F_{n+1}=F_{n} A \vee\left(A^{n} \wedge\left(F_{n+1}: E\right)\right) E=F_{n} A$ $\vee\left(A^{n-1} \wedge\left(F_{n}: E\right)\right) A E=F_{n} A$, which again contradicts the assumption that $F \in \mathscr{F}$ and shows that $\mathscr{F}=\varnothing$. Q.E.D.

COROLlaRY 2.9. If $A$ and $B$ are elements of $\mathscr{L}$, then there exists an integer $N$ such that $B \wedge A^{N+n}=\left(B \wedge A^{N}\right) A^{n}$, for all $n \geqq 0$.

LEMma 2.10. If $C \in \mathscr{R}(\mathscr{L}, A)$, then $C$ is the join of finitely many principal elements of the form $B^{(r)}$ where $B \leqq A^{r}$ is principal in $\mathscr{L}$.

Proof. Choose integers $N$ and $K(K \leqq N)$ such that $C_{N+i}=C_{N} A^{i}$, for $i \geqq 0$, and $C_{K+i}=C_{K}^{\prime}$, for $i \leqq 0$. Then clearly $C=\bigvee_{K \leqq i \leqq N}\left(C_{i}\right)^{(i)}$. For each $i, K \leqq i \leqq N$, choose finitely many principal elements $B_{i, j}$ in $\mathscr{L}$ such that $\bigvee_{j} B_{i, j}=C_{i}$. Then

$$
C=\bigvee_{i, j}\left(B_{i, j}\right)^{(i)}
$$

THEOREM 2.11. $\mathscr{R}(\mathscr{L}, A)$ is a Noether lattice.

Proof. By Lemmas 2.1 and 2.10 , it is only necessary to show that $\mathscr{R}(\mathscr{L}, A)$ satisfies the ascending chain condition. Hence, let $F(1) \leqq F(2) \leqq \cdots$ be an ascending chain in $\mathscr{R}(\mathscr{L}, A)$. Set $S=\bigvee_{j} F(j)$ so that, for each $i, S_{i}=\bigvee_{j}(F(j))_{i}$. Choose integers $N$ and $K(K \leqq N)$ so that $S_{N+i}=S_{N} A^{i}$, for $i \geqq 0$, and $S_{K+i}=S_{K}$, for $i \leqq 0$. For 
each $i, K \leqq i \leqq N$, choose $F(\varnothing(i))$ so that $(F(\varnothing(i)))_{i}=S_{i}$; and let $M$ be the maximum of the elements $F(\varnothing(i))$ thus chosen. Then, as in the proof of Theorem 2.8, $S=M$ $=F(j)$, for some $j$. Q.E.D.

It is natural to define the altitude of a Noether lattice $\mathscr{L}$ to be the supremum of the heights of the prime elements $P$ of $\mathscr{L}(P<I)$. As for rings, the altitude of $\mathscr{L}$ will be denoted by alt $\mathscr{L}$.

The altitude of $\mathscr{R}(\mathscr{L}, A)$ will be determined presently in terms of the altitude of $\mathscr{L}$ and certain properties of $A$. However, before doing this, it is convenient first to investigate some of the basic properties of $\mathscr{R}(\mathscr{L}, A)$.

If $B$ is any element of $\mathscr{L}$, then it is clear that for every $i, A^{i} \geqq B \wedge A^{i} \geqq B \wedge A^{i+1}$ $\geqq\left(A\left(B \wedge A^{i}\right)\right.$, so that $\sum\left(B \wedge A_{i}\right)$ is an element of $\mathscr{R}(\mathscr{L}, A)$. This element will be denoted by $B^{*}$.

Lemma 2.12. The correspondence $B \rightarrow B^{*}$ of $\mathscr{L}$ into $\mathscr{R}(\mathscr{L}, A)$ is $1-1$, isotone and meet-preserving. If $P \in \mathscr{L}$ is prime and $Q \in \mathscr{L}$ is $P$-Primary, then $Q^{*}$ is $P^{*}$-primary in $\mathscr{R}(\mathscr{L} A)$.

Proof. The first statement is immediate from the definition of $B^{*}$. Hence assume that $P$ is a prime element of $\mathscr{L}$ and that $Q$ is $P$-primary. If $D$ and $E$ are any elements of $\mathscr{R}(\mathscr{L}, A)$ with $D E \leqq Q^{*}$ and $D \$ Q^{*}$, then there exists an integer $s$ such that $D_{s} \ Q \wedge A^{s}$ and therefore also such that $D_{s} \$$. Now, for every choice of $r$, $D_{s} E_{r} \leqq(D E)_{s+r} \leqq Q \wedge A^{s+r} \leqq Q$, so it follows from the assumption that $Q$ is $P$ primary in $\mathscr{L}$ that $E_{\mathrm{r}} \leqq P$, for all $r$, so that $E_{r} \leqq P \wedge A^{r}$, for all $r$. Hence $E \leqq P^{*}$. Now, choose $n$ so that $P^{n} \leqq Q \leqq P$ in $\mathscr{L}$. It is easily seen that for every $r$ and $s,\left(B \wedge A^{r}\right)$ $\cdot\left(C \wedge A^{s}\right) \leqq B C \wedge A^{r+s}$, and it follows from this that $B^{*} C^{*} \leqq(B C)^{*}$. Hence, in particular, $\left(P^{*}\right)^{n} \leqq\left(P^{n}\right)^{*}$ and therefore $\left(P^{*}\right)^{n} \leqq\left(P^{n}\right)^{*} \leqq Q^{*} \leqq P^{*}$, so that [1] $P^{*}$ is prime and $Q^{*}$ is $P^{*}$-primary. Q.E.D.

Corollary 2.13. Let $B=Q_{1} \wedge \cdots \wedge Q_{K}$ be a normal decomposition of $B$ in $\mathscr{L}$ in which $Q_{i}$ is $P_{i}$-primary. Then $B^{*}=Q_{1}^{*} \wedge \cdots \wedge Q_{K}^{*}$ is a normal decomposition of $B^{*}$ in $\mathscr{R}(\mathscr{L}, A)$ in which $Q_{1}^{*}$ is $P_{i}^{*}$-primary. Furthermore, if $P_{1}, \ldots, P_{r}$ are the minimal associated primes of $B$, then $P_{1}^{*}, \ldots, P_{r}^{*}$ are the minimal associated primes of $B^{*}$.

Proof. This is immediate from Lemma 2.12. Q.E.D.

Lemma 2.14. Let $P$ be a prime element of $\mathscr{R}(\mathscr{L}, A)$ with $I^{(-1)} P$. Then $P=\left(P_{0}\right)^{*}$.

Proof. Assume $I^{(-1)} P$. Choose $s$ so that $P_{0} \wedge A^{s+i}=\left(P_{0} \wedge A^{s}\right) A^{i}$ for all $i \geqq 0$ (Corollary 2.9). Then $\left(P_{0}\right)^{*} I^{(-s)}=P_{0}^{*}\left(I^{(-1)}\right)^{s} \leqq P$ (Corollary 2.5$)$, so $\left(P_{0}\right)^{*} \leqq P$. Now, if $i \geqq 0$, then $P_{i} \leqq P_{0} \wedge A^{i}$; and if $i<0$, then $\left(P_{i}\right)^{(0)} I^{(i)}=\left(P_{i}\right)^{(0)}\left(I^{(-1)}\right)^{-i} \leqq P$, so that $P_{i} \leqq P_{0}$. It follows therefore that also $P \leqq P_{0}^{*}$ and hence that $P=\left(P_{0}\right)^{*}$. Q.E.D.

Lemma 2.15. Let $P$ denote a maximal element of $\mathscr{L}$. If $P \geqq A$, then $P^{*} \vee I^{(-1)}$ is maximal in $\mathscr{R}(\mathscr{L}, A)$. If $P \geq A$, then $P^{*}$ is maximal in $\mathscr{R}(\mathscr{L}, A)$. Furthermore, if $M$ is any maximal element of $\mathscr{R}(\mathscr{L}, A)$, then $M_{0}$ is maximal in $\mathscr{L}$ and either $M=\left(M_{0}\right)^{*} \vee I^{(-1)}$ or $M=\left(M_{0}\right)^{*}$, depending on whether $A \leqq M_{0}$ or $A \leqq M_{0}$. 
Proof. It is easily seen that if an element $M$ of $\mathscr{R}(\mathscr{L}, A)$ is maximal, then the conditions $M \geqq I^{(-1)}$ and $M_{0} \geqq A$ are equivalent. Note that if $B \in \mathscr{R}(\mathscr{L}, A)$, then $B_{0}=I$ if, and only if, $B=I^{*}$. Now, assume that $P$ is maximal in $\mathscr{L}$ and that $P \geq A$. Then $\left(P^{*} \vee I^{(-1)}\right)_{0}=P \vee A=I$, so that if $P^{*} \leqq T<I^{*}$, then also $I^{(-1)} \leq T$. Furthermore, if $P^{*} \leqq T<I^{*}$, then $P=\left(P^{*}\right)_{0}=T_{0}$, so that, if $T$ is maximal, then (Lemma 2.14) $T=\left(T_{0}\right)^{*}=P^{*}$. Assume now that $P \geqq A$. Then $\left(P^{*} \vee I^{(-1)}\right)_{0}=P$, so $P^{*} \vee I^{(-1)}<I^{*}$, and $\left(P^{*} \vee I^{(-1)}\right)_{i}=A_{i}$ for all $i \neq 0$, so that $P^{*} \vee I^{(-1)}$ is clearly maximal in $\mathscr{R}(\mathscr{L}, A)$.

Assume now that $M$ is maximal in $\mathscr{R}(\mathscr{L}, A)$. If $M_{0} \geqq A$, then $M \geqq I^{(-1)}$ and $M_{i}=A^{i}$ for all $i \neq 0$, so that $M=\left(M_{0}\right)^{*} \vee I^{(-1)}$. If $M_{0}$ were not maximal in $\mathscr{L}$, say $M_{0}<P<I$, then the inequality $M=\left(M_{0}\right)^{*} \vee I^{(-1)}<P^{*} \vee I^{(-1)}<I^{*}$ would hold in $\mathscr{R}(\mathscr{L}, A)$, in contradiction to the maximality of $M$. If $M_{0} \neq A$, then $M \geq I^{(-1)}$ and $M=M_{0}^{*}$, by Lemma 2.14 . In this case, $M_{0}$ is maximal in $\mathscr{L}$ since $M_{0}<T$ would imply $\left(M_{0}\right)^{*}$ $<T^{*}$. Q.E.D.

If $P$ is a maximal element of $\mathscr{L}$, denote by $m(P)$ the associated maximal element of $\mathscr{R}(\mathscr{L}, A)$.

Lemma 2.16. Let $P$ be a maximal element of $\mathscr{L}$. Then ht $m(P)=$ ht $P+1$ if $P \geqq A$ and ht $m(P)=$ ht $P$ if $P \geq A$.

Proof. Let $P_{0}<P_{1}<\cdots<P_{d}=P$ be a prime chain in $\mathscr{L}(d=$ ht $P)$. Then in $\mathscr{R}(\mathscr{L}, A), P_{0}^{*}<P_{1}^{*}<\cdots<P_{d}^{*}<P^{*} \vee I^{(-1)} \leqq I$, with the equality $P^{*} \vee I^{(-1)}=I$ holding if, and only if, $P \geq A$. It follows that ht $m(P) \geqq$ ht $P$ and that ht $m(P) \geqq$ ht $P+1$ if $P \geqq A$.

Now, if $m(P)=P^{*}$, then it follows from Lemma 2.14 that any prime chain for $m(P)$ is the image under the map $B \rightarrow B^{*}$ of a prime chain of the same length for $P$ in $\mathscr{L}$, and hence that ht $m(P) \leqq$ ht $P$. It can be assumed therefore that $m(P) \neq P^{*}$, so that (Lemmas 2.14 and 2.15) $I^{(-1)} \leqq m(P)$ and $P \geqq A$. Now, let $A_{1}, \cdots, A_{s}$ be principal elements of $\mathscr{L}$ with $A=A_{1} \vee \cdots \vee A_{s}$. The inequality ht $m(P) \leqq$ ht $P+1$ will now be established by induction on $s$. Hence assume $s=1$, and let $P(0)<P(1)$ $<\cdots<P(d+i)=m(P)$ be a prime chain in $\mathscr{R}(\mathscr{L}, A)$ with $d=$ ht $P$ and $d+i=$ ht $m(P)$. If $A^{(1)} \leqq P(0)$, then by Theorem 2.7 above and Lemma 6.4 in [1], it can be assumed that $I^{(-1)} \leqq P(1)$ (be rechoosing $P(1)$ if necessary). Then $P(1)_{0}<\cdots<P(d+i)_{0}=P$ is a prime chain in $\mathscr{L}$; so, in this case, $d+i-1 \leqq d$. If, on the other hand, $A^{(1)} P(0)$ it can be assumed that $A^{(1)} \leqq P(1)$. Now, if also $I^{(-1)} \leqq P(1)$ then $P(0)_{0}<P(1)_{0}<\cdots$ $<P(d+i)_{0}=P$ is a prime chain in $\mathscr{L}$, in contradiction to the assumption that ht $P=d$. Hence, $I^{(-1)} \ P(1)$, so $d+i \geqq 2$; and it can be assumed that $I^{(-1)} \leqq P(2)$. Then $P(0)_{0}<P(2)_{0}<\cdots<P(d+i)_{0}=P$ is a prime chain in $\mathscr{L}$, so again $d+i-1 \leqq d$. Therefore, if $s=1$, then ht $m(P) \leqq$ ht $P+1$. Assume now that $s>1$. If $A_{j}{ }^{1)} \leqq P(0)$, for all $j$, then, as before (by rechoosing $P(1)$ if necessary), it can be assumed that $I^{(-1)} \leqq P(1)$, so that $P(0)_{0}<P(2)_{0}<\cdots<P(d+i)_{0}=P$ in $\mathscr{L}$; and therefore $d+i-1 \leqq d$. Assume, therefore, that $A_{1}^{(1)} \$ P(0)$. Then, as above, it can also be assumed that $A_{1}^{(1)} \leqq P(1)$; and by continuing, it can be assumed that $A_{j}^{(1)} \leqq P(h)$, for all $j$, and that for $1 \leqq j \leqq h$, $A_{j}^{(1)} \leqq P(j)$ and $A_{j}^{(1)} \leqq P(j-1)$. Also, it can be assumed that $I^{(-1)} \leqq P(r), I^{(-1)}$ 
$\$ P(r-1)$, and that $1 \leqq r \leqq h+1$. Now, set $B=\bigvee_{j \neq 1} A_{j}$. Then the elements $P_{j}^{\prime}$ $=\sum\left(P(j)_{i} \wedge B^{i}\right)$ of $\mathscr{R}(\mathscr{L}, B)$ are prime $(j=0, \ldots, d+i)$. To see this, assume $C$ and $D$ are elements of $\mathscr{L}$ with $C \leqq B^{r}, D \leqq B^{s}$ and $C_{B}^{(r)} D_{B}^{(s)}=(C D)_{B}^{(r+s)} \leqq P_{j}^{\prime}$. Then $C D \leqq\left(P_{j}^{\prime}\right)_{r+s}=P(j)_{r+s} \wedge B^{r+s}$, so $(C D)_{A}^{(r+s)}=C_{A}^{(r)} D_{A}^{(s)} \leqq P(j)$ and therefore either $C_{A}^{(r)} \leqq P(j)$ or $D_{A}^{(s)} \leqq P(j)$. Assume $C_{A}^{(r)} \leqq P(j)$. Then $C \leqq P(j)_{r}$ and therefore $C \leqq P(j)_{r}$ $\wedge B^{r}=\left(P_{j}^{\prime}\right)_{r}$, so $C_{B}^{(r)} \leqq P_{j}^{\prime}$. By Lemma 2.10 it now follows that $P_{j}^{\prime}$ is prime. Further, $P_{d+1}^{\prime}$ is the maximal element $m(P)$ determined in $\mathscr{R}(\mathscr{L}, B)$ by $P$. It will now be shown that also $P_{0}^{\prime}<P_{1}^{\prime}<\cdots<P_{d+1}^{\prime}$. Since either $P(1) \geqq I^{(-1)}$ and $P(0) \geq I^{(-1)}$ or else $P(1)$ and $P(0)$ are the images of distinct primes in $\mathscr{L}$ under the map $C \rightarrow C^{*}$ of $\mathscr{L}$ into $\mathscr{R}(\mathscr{L}, A)$, it follows that $P_{0}^{\prime} \neq P_{1}^{\prime}$. Also $P_{j}^{\prime} \neq P_{j+1}^{\prime}$ for $1 \leqq j \leqq h-1$, since, in this case, $A_{j+1} \leqq B, A_{j+1}^{(1)} \leqq P(j+1)$, and $A_{j+1}^{(1)} P(j)$, so that $P(j)_{1} \wedge B \neq P(j+1)_{1} \wedge B$. Now, $P_{h}^{\prime} \neq P_{h+1}^{\prime}$, since either $P(h+1) \geqq I^{(-1)}$ and $P(h) \geq I^{(-1)}$, or else $P(h+1)_{0}$ $\neq P(h)_{0}$. And $P_{j}^{\prime} \neq P_{j+1}^{\prime}$ for $j>h$, since necessarily $P(j)_{0} \neq P(j+1)_{0}$.

Hence, $P_{0}^{\prime}<P_{1}^{\prime}<\cdots<P_{d+i}^{\prime}=m(p)$ is a prime chain in $\mathscr{R}(\mathscr{L}, B)$; so by the inductive hypothesis, $d+i \leqq d+1$. Therefore, ht $m(p) \leqq$ ht $P+1$. Q.E.D.

These results are summarized for local lattices by the following

THeOREM 2.17. If $\mathscr{L}$ is a local Noether latice of altitude $d$, and if $A \in \mathscr{L}$ is contained in the maximal element, then $\mathscr{R}(\mathscr{L}, A)$ is a local Noether lattice of altitude $d+1$.

3. Hereafter $\mathscr{L}$ will denote a local Noether lattice with maximal element $P$.

In this section it will be shown that if $Q$ is an element of $\mathscr{L}$ which is $P$-primary, then there exists a polynomial $D^{*}(Q, x)$ such that for all $n$ sufficiently large, $D^{*}(Q, n)$ is the lattice dimension of the quotient $I / Q^{n}$. Also, it will be shown that, if $A$ is any element of $\mathscr{L}$, then there exists a polynomial $B^{*}(A, x)$ such that for all sufficiently large $n, B^{*}(A, n)$ is the number of elements in a minimal base for $A^{n}$.

Lemma 3.1. Let $M$ denote the maximal element of $\mathscr{R}(\mathscr{L}, A)$, and let $B$ be an element of $\mathscr{R}(\mathscr{L}, A)$, such that $M_{1} / B_{1}$ is finite dimensional. Then $M_{n} / B_{n}$ is finite dimensional for all $n \geqq 1$.

Proof. Since $M_{n}=A^{n} \geqq B_{n} \geqq B_{1} A^{n-1}$, it suffices to show the finite dimensionality of $A^{n} / B_{1} A^{n-1}$, for $n \geqq 2$. Let $C_{1}, \ldots, C_{K}$ be elements of $\mathscr{L}$ such that $B_{1}=C_{1}<C_{2}$ $<\cdots<C_{k}=A$, and such that for each $i=1, \ldots, K-1, C_{i+1}$ is a cover for $C_{i}$. Then $A^{n-1} B_{1}=A^{n-1} C_{1} \leqq A^{n-1} C_{2} \leqq \cdots \leqq A^{n-1} C_{K}=A^{n}$, so it suffices to show that each of the quotients $A^{n-1} C_{i+1} / A^{n-1} C_{i}$ is finite dimensional. Let $i$ be fixed, $1 \leqq i \leqq K-1$. Then by Theorem 1.2, there exists a principal element $C \in \mathscr{L}$ with $C_{i} \vee C=C_{i+1}$ and $C_{i}: C=P$. Let $A_{1}, \ldots, A_{r}$ be a basis for $A^{n-1}$. Then

$$
A^{n-1} C_{i} \leqq A^{n-1} C_{i} \vee A_{1} C \leqq \cdots \leqq A^{n-1} C_{i} \vee A_{1} C \vee \cdots \vee A_{r} C=A^{n-1} C_{i+1}
$$

and

$$
P\left(A^{n-1} C_{i} \vee A_{1} C \vee \cdots \vee A_{j+1} C\right) \leqq\left(A^{n-1} C_{i} \vee A_{1} C \vee \cdots \vee A_{j} C\right)
$$

for $j=1, \ldots, r-1$, so that (Theorem 1.2) there exists a composition chain for the quotient $A^{n-1} C_{i+1} / A^{n-1} C_{i}$. Q.E.D. 
THEOREM 3.2. Let $M$ and $B$ be as in Lemma 3.1. Then there exists a polynomial $p(x)$ with rational coefficients such that for all $n$ sufficiently large, $p(n)$ is the lattice dimension $d\left(M_{n} / B_{n}\right)$ of the quotient $M_{n} / B_{n}$.

Proof. Let $\mathscr{F}$ be the family of all elements $B \in \mathscr{R}(\mathscr{L}, A)$ for which $M_{1} / B_{1}$ is finite dimensional and for which there exists no polynomial with the desired properties. If $\mathscr{F} \neq \varnothing$, then $\mathscr{F}$ has a maximal element $C$, since $\mathscr{R}(\mathscr{L}, A)$ is Noetherian. Clearly, $C_{1}<M_{1}$, so there exists a principal element $E \in \mathscr{L}$ with $C<C$ $\vee E^{(1)} \leqq M$. Then by the choice of $C$ and $E, C \vee E^{(1)} \notin \mathscr{F}$ and there exists a polynomial $p^{\prime}(x)$ for $C \vee E^{(1)}$. Set $D=C: E^{(1)}$ so that (Lemma 2.6) $D_{n}=\left(C_{n+1}: E\right) \wedge A^{n}$, for all $n$. Then $D \geqq C$, and

$$
\begin{aligned}
d\left(M_{n} /\left(C \vee E^{(1)}\right)_{n}\right) & =d\left(M_{n} /\left(C_{n} \vee E A^{n-1}\right)\right) \\
& =d\left(M_{n} / C_{n}\right)-d\left(\left(C_{n} \vee E A^{n-1}\right) / C_{n}\right) \\
& =d\left(M_{n} / C_{n}\right)-d\left(E A^{n-1} /\left(C_{n} \wedge E A^{n-1}\right)\right) \\
& =d\left(M_{n} / C_{n}\right)-d\left(A^{n-1} /\left(C_{n}: E\right) \wedge A^{n-1}\right) \\
& =d\left(M_{n} / C_{n}\right)-d\left(M_{n-1} / D_{n-1}\right),
\end{aligned}
$$

by Lemma 1.1. Now, if $D>C$, then also $D \notin \mathscr{F}$ and there exists a polynomial $p^{\prime \prime}(x)$ for $D$. But then

$$
\begin{aligned}
d\left(M_{n} / C_{n}\right) & =d\left(M_{n} /\left(C \vee E^{(1)}\right)_{n}\right)+d\left(M_{n-1} / D_{n-1}\right) \\
& =p^{\prime}(n)+p^{\prime \prime}(n-1)
\end{aligned}
$$

for all $n$ sufficiently large, which is clearly a contradiction. Hence $D=C$, so $D_{n}$ $=\left(C_{n+1}: E\right) \wedge A^{n}=C_{n}$ and $d\left(M_{n} /\left(C \vee E^{(1)}\right)_{n}\right)=d\left(M_{n} / C_{n}\right)-d\left(M_{n-1} / C_{n-1}\right)$ for all $n$. Choose $N$ so that $d\left(M_{n} /\left(C \vee E^{(1)}\right)_{n}\right)=p^{\prime}(n)$ for $n \geqq N$. Then

$$
\sum_{n=N}^{w} p^{\prime}(n)=d\left(M_{w} / C_{w}\right)-d\left(M_{N-1} / C_{N-1}\right) \text { for } w>N,
$$

so

$$
d\left(M_{w} / C_{w}\right)=\sum_{n=N}^{w} p^{\prime}(n)+d\left(M_{N-1} / C_{N-1}\right) \quad \text { for } w>N
$$

But this again contradicts the choice of $C$ and hence shows that $\mathscr{F}=\varnothing$. Q.E.D.

Before proceeding further, it is convenient to introduce the following notation:

(3.1) If $C \in \mathscr{L}$, then for $n \geqq 1, B(C, n)$ is the number of elements in a minimal base for $C^{n}$.

(3.2) If $Q$ is a $P$-primary element of $\mathscr{L}$, then for $n \geqq 0, F(Q, n)$ is the dimension of the quotient $Q^{n} / Q^{n+1}$.

(3.3) If $Q$ is a $P$-primary element of $\mathscr{L}$, then for $n \geqq 1, D(Q, n)$ is the dimension of the quotient $I / Q^{n}$.

Corollary 3.3. Let $C$ be an element of $\mathscr{L}$. Then there exists a polynomial $B^{*}(C, X)$ such that $B^{*}(C, n)=B(C, n)$ for all $n$ sufficiently large. 
Proof. Set $B=(P C)^{(1)}$ in $\mathscr{R}(\mathscr{L}, C)$. Then $B_{n}=P C^{n}$ for $n \geqq 1$ and $d\left(M_{n} / B_{n}\right)$ $=d\left(C^{n} / P C^{n}\right)=B(C, n)$, by Theorem 1.4. The Corollary now follows from Theorem 3.2. Q.E.D.

Corollary 3.4. Let $Q$ be a P-primary element of $\mathscr{L}$. Then there exists a polynomial $F^{*}(Q, X)$ such that $F^{*}(Q, n)=F(Q, n)$ for all $n$ sufficiently large.

Proof. Set $B=Q^{(0)}$ in $\mathscr{R}(\mathscr{L}, Q)$. Q.E.D.

Corollary 3.5. Let $Q$ be a P-primary element of $\mathscr{L}$. Then there exists a polynomial $D^{*}(Q, X)$ such that $D^{*}(Q, n)=D(Q, n)$ for all $n$ sufficiently large.

Proof. This is immediate from Corollary 3.4. Q.E.D:

Let $\partial p(x)$ denote the degree of a polynomial $p(x)$. It will presently be shown that if $\mathscr{L}$ has altitude $d$, then for every choice of the $P$-primary element $Q, \partial B^{*}(Q, X)$ $=\partial F^{*}(Q, X)=d-1$ and $\partial D^{*}(Q, X)=d$. Of course, the equality $\partial D^{*}(Q, X)=$ $\partial F^{*}(Q, X)+1$ is clear, since for all sufficiently large $n, D^{*}(Q, n+1)-D^{*}(Q, n)=$ $F^{*}(Q, n)$. And since $P^{K} \leqq Q \leqq P$ for some $K$, it is immediate that $\partial D^{*}(Q, X)$ $=\partial D^{*}(P, X)$ and hence also that $\partial F^{*}(Q, X)=\partial F^{*}(P, X)$. These remarks establish part of the following

LEMMA 3.6. If $Q$ is a P-primary element, then

$$
\begin{aligned}
\partial B^{*}(Q, X) & =\partial B^{*}(P, X)=\partial F^{*}(Q, X)=\partial F^{*}(P, X) \\
& =\partial D^{*}(Q, X)-1=\partial D^{*}(P, X)-1 .
\end{aligned}
$$

Proof. Since $B^{*}(P, X)=F^{*}(P, X)$, it is necessary only to show that $\partial B^{*}(Q, X)$ $=\partial F^{*}(Q, X)$. Choose $K$ so that $P^{K} \leqq Q$ and let $h=B(P, 1)$. Then

$$
\begin{aligned}
F(Q, n) & =d\left(Q^{n} / Q^{n+1}\right)=d\left(Q^{n} / Q^{n} P\right)+d\left(Q^{n} P / Q^{n+1}\right) \\
& \leqq d\left(Q^{n} / Q^{n} P\right)+d\left(Q^{n} P / Q^{n} P^{K}\right)=d\left(Q^{n} / Q^{n} P\right)+\sum_{r=1}^{K-1} d\left(Q^{n} P^{r} / Q^{n} P^{r+1}\right) \\
& \leqq d\left(Q^{n} / Q^{n} P\right)+\sum_{r=1}^{K-1} d\left(Q^{n} / Q^{n} P\right) h^{r} \leqq B(Q, n)+B(Q, n)(K-1) h^{(K-1)} \\
& =B(Q, n)\left(1+(K-1) h^{(K-1)}\right) .
\end{aligned}
$$

Since also $B(Q, n) \leqq F(Q, n)$, it follows that $\partial B^{*}(Q, X) \leqq \partial F^{*}(Q, X) \leqq \partial B^{*}(Q, X)$. Q.E.D.

Corollary 3.7. If $Q$ is a P-primary element of $\mathscr{L}$ with $B(Q, 1)=h$, then $\partial D^{*}(Q, X) \leqq h$.

Proof. If $B(Q, 1)=h$, then clearly

$$
B(Q, n) \leqq\left(\begin{array}{c}
n+h-1 \\
h-1
\end{array}\right)
$$

It follows therefore that $\partial B^{*}(Q, X)=\partial D^{*}(Q, X)-1 \leqq h-1$. Q.E.D. 
Lemma 3.8. If $Q$ is a P-primary element of $\mathscr{L}$, then $\partial D^{*}(Q, X) \geqq$ alt $\mathscr{L}$.

Proof. By Lemma 3.6, it is sufficient to show that $\partial D^{*}(P, X) \geqq$ alt $\mathscr{L}$. If alt $\mathscr{L}=0$, then $P^{n}=0$ for all large $n$, so $\partial D^{*}(P, X)=0$. Hence assume $\mathscr{L}$ has altitude $d>0$ and let $P_{0}<P_{1}<\cdots<P_{d}=P$ be a prime chain in $\mathscr{L}$. Then alt $\mathscr{L} \mid P_{0}=$ alt $\mathscr{L}$ and $D^{*}\left(P / P_{0}, X\right) \leqq D^{*}(P, X)$. Let $A \leqq P$ be a principal element of $\mathscr{L}$ with $A \npreceq P_{0}$ so that $A / P_{0}$ is principal in $\mathscr{L} \mid P_{0}$ with $A \mid P_{0} \neq 0$. Choose $K$ so that

$$
\left(P / P_{0}\right)^{K+n} \wedge\left(A / P_{0}\right)=\left[\left(P_{0} / P_{0}\right)^{K} \wedge\left(A / P_{0}\right)\right]\left(P / P_{0}\right)^{n}, \text { for all } n \geqq 0
$$

(Corollary 2.9). Then

$$
\left(\left(P / P_{0}\right)^{K+n}:\left(A / P_{0}\right)\right)\left(A / P_{0}\right)=\left(\left(P / P_{0}\right)^{K}:\left(A / P_{0}\right)\right)\left(P / P_{0}\right)^{n}\left(A / P_{0}\right)
$$

and hence

$$
\left(P / P_{0}\right)^{K+n}:\left(A / P_{0}\right)=\left(\left(P / P_{0}\right)^{K}:\left(A / P_{0}\right)\right)\left(P / P_{0}\right)^{n} \leqq\left(P / P_{0}\right)^{n},
$$

for all $n \leqq 0$ (Lemma 1.1). Now, since $A \leqq P,\left(P / P_{0}\right)^{K+n-1} \leqq\left(\left(P / P_{0}\right)^{K+n}: A / P_{0}\right)$. It follows that

$$
\begin{aligned}
D\left(P / P_{0}, K+n-1\right) & \geqq \operatorname{dim}\left(I\left[\left(P / P_{0}\right)^{K+n}:\left(A / P_{0}\right)\right]\right) \\
& =\operatorname{dim}\left(\left(A / P_{0}\right) /\left[\left(\left(P / P_{0}\right)^{K+n}:\left(A / P_{0}\right)\right)\left(A / P_{0}\right)\right]\right) \\
& =\operatorname{dim}\left(\left(A / P_{0}\right) /\left(P / P_{0}\right)^{K+n} \wedge\left(A / P_{0}\right)\right) \\
& =\operatorname{dim}\left(\left(\left(P / P_{0}\right)^{n+K} \vee A\right) /\left(P / P_{0}\right)^{n+K}\right) \\
& =D\left(P / P_{0}, n+K\right)-D\left(P /\left(P_{0} \vee A\right), n+K\right) \geqq D\left(P / P_{0}, n\right),
\end{aligned}
$$

so

$$
\begin{aligned}
D\left(P / P_{0}, n+K-1\right) & \geqq D\left(P / P_{0}, n+K\right)-D\left(P /\left(P_{0} \vee A\right), n+k\right) \\
& \geqq D\left(P / P_{0}, n\right) \text { for all } n \geqq 0 .
\end{aligned}
$$

Hence for $n$ sufficiently large,

$$
D^{*}\left(P /\left(P_{0} \vee A\right), n+K\right) \geqq D^{*}\left(P / P_{0}, n+K\right)-D^{*}\left(P / P_{0}, n+K-1\right)
$$

and

$$
D^{*}\left(P / P_{0}, n+K\right)-D^{*}\left(P / P_{0}, n\right) \geqq D^{*}\left(P /\left(P_{0} \vee A\right), n+K\right),
$$

so $\partial D^{*}\left(P /\left(P_{0} \vee A\right), X\right)=\partial D^{*}\left(P / P_{0}, X\right)-1$. Now, it is clear that alt $\mathscr{L} /\left(P_{0} \vee A\right)$ $\leqq d-1$ and therefore (Lemma 1.8) that alt $\mathscr{L} /\left(P_{0} \vee A\right)=d-1$. Hence, by the inductive hypothesis, $\partial D^{*}\left(P / P_{0} \vee A\right) \geqq d-1$ so that

$$
\begin{aligned}
\partial D^{*}(P, X) & \geqq \partial D^{*}\left(P / P_{0}, X\right)=1+\partial D^{*}\left(P /\left(P_{0} \vee A\right), X\right) \\
& \geqq \text { alt } \mathscr{L} /\left(P_{0} \vee A\right)+1=\text { alt } \mathscr{L} / P_{0}=\text { alt } \mathscr{L} . \quad \text { Q.E.D. }
\end{aligned}
$$

TheOREM 3.9. If $Q$ is a P-primary element of the local lattice $\mathscr{L}$, then

$$
\partial D^{*}(Q, X)=\text { alt } \mathscr{L} \text {. }
$$


Proof. Let $A \neq 0$ be a principal element of $\mathscr{L}$ with $A \leqq Q$. Choose $s$ so that $Q^{n+s} \wedge A=Q^{n}\left(Q^{s} \wedge A\right)$, for all $n \geqq 0$. Then $\left(Q^{n+s}: A\right) A \leqq Q^{n} A$, so $Q^{n+s}: A \leqq Q^{n}$ $\vee(0: A)$, since $A$ is principal. Now,

$$
\begin{aligned}
\left.\operatorname{dim}\left(I / Q^{n} \vee A\right)\right) & =\operatorname{dim}\left(I / Q^{n}\right)-\operatorname{dim}\left(Q^{n} \vee A / Q^{n}\right) \\
& =\operatorname{dim}\left(I / Q^{n}\right)-\operatorname{dim}\left(A / Q^{n} \wedge A\right) \\
& =\operatorname{dim}\left(I / Q^{n}\right)-\operatorname{dim}\left(I / Q^{n}: A\right)
\end{aligned}
$$

so

$$
\operatorname{dim}\left(I / Q^{n}\right)-\operatorname{dim}\left(I / Q^{n-1} \vee(0: A)\right) \leqq \operatorname{dim}\left(I / Q^{n} \vee A\right)
$$

and

$$
\operatorname{dim}\left(I / Q^{n} \vee A\right) \leqq \operatorname{dim}\left(I / Q^{n}\right)-\operatorname{dim}\left(I / Q^{n-s} \vee(0: A)\right)
$$

Hence

$$
D(Q, n)-D(Q /(0: A), n-1) \leqq D(Q / A, n)
$$

and

$$
D(Q / A, n) \leqq D(Q, n)-D(Q /(0: A), n-s)
$$

so that

$$
D^{*}(Q, n) \leqq D^{*}(Q / A, n)+D^{*}(Q /(0: A), n-1)
$$

and

$$
D^{*}(Q, n) \geqq D^{*}(Q / A, n)+D^{*}(Q /(0: A), n-s),
$$

for all $n$ sufficiently large. It follows that one of the polynomials $D^{*}(Q / A, X)$ and $D^{*}(Q /(0: A), X)$ has the same degree as $D^{*}(Q, X)$.

Now, let $A_{1}, \ldots, A_{h}$ be principal elements in $\mathscr{L}$ with $Q=A_{1} \vee \cdots \vee A_{h}$; and set $d=$ alt $\mathscr{L}$. By Lemma 3.6 it can be assumed that no fewer than $h$ principal elements have join a $P$-primary element. If $d=0$, then the calculation is immediate, so assume $d>0$. Choose $K$ so that $0:\left(A_{h}\right)^{K}=0:\left(A_{h}\right)^{K+i}$ for all $i \geqq 0$, and set $A=\left(A_{h}\right)^{K}$. Then $A_{1} \vee \cdots \vee A_{h-1} \vee A$ is $P$-primary element of $\mathscr{L}$, so (Lemma 3.6) it can be assumed $Q=A_{1} \vee \cdots \vee A_{h-1} \vee A$. Now, if $h-d=0$, then (Corollary 3.7 and Lemma 3.8) $d \leqq \partial D^{*}(Q, X) \leqq h=d$. Hence it can be assumed $h-d>0$.

Now assume, $\partial D^{*}(Q, X)=\partial D^{*}(Q \mid A, X)$. If alt $\mathscr{L} \mid A<d$, then it follows by the induction on altitude that $\partial D^{*}(Q \mid A, X)=$ alt $\mathscr{L} \mid A$ so that $\partial D^{*}(Q, X)=$ $\partial D^{*}(Q \mid A, X) \leqq d$. On the other hand, if alt $\mathscr{L} \mid A=d$, then it follows by the induction on the number of generators that $\partial D^{*}(Q / A, X)=$ alt $\mathscr{L} \mid A$ and hence that $\partial D^{*}(Q, X)=d$. Hence if $\partial D^{*}(Q, X)=\partial D^{*}(Q / A, X)$, then $\partial D^{*}(Q, X)=d$.

Assume now that $\partial D^{*}(Q, X)=\partial D^{*}(Q /(0: A), X)$. By the choice of $A, A /(0: A)$ is not a zero divisor in $\mathscr{L} /(0: A)$. Hence, by the earlier part of the proof,

$$
D^{*}(Q /(0: A), n) \leqq D^{*}(Q /(0: A) \vee A, n)+D^{*}(Q /(0: A), n-1)
$$


and

$$
D^{*}(Q /(0: A), n) \geqq D^{*}(Q /(0: A) \vee A, n)+D^{*}(Q /(0: A), n-s)
$$

so

$$
c D^{*}(Q /(0: A) \vee A, X)=c D^{*}(Q /(0: A), X)-1=\partial D^{*}(Q, X)-1 .
$$

It is easily seen that $\mathscr{L} /(0: A) \vee A$ has altitude $<d$, so it follows by the inductive assumption on altitude that

$$
D^{*}(Q /(0: A) \vee A, X)=\dot{C} D^{*}(Q, X)-1<d,
$$

and hence (Lemma 3.8) that $c D^{*}(Q, X)=d$. Q.E.D.

4. Regular local lattices. A local lattice $\mathscr{L}$ of altitude $d$ is said to be a regular local lattice if the maximal element $P \in \mathscr{L}$ is the join of $d$-principal elements.

It is noted that by Theorem 1.4 , the local lattice $\mathscr{L}$ is regular if, and only if, $\operatorname{dim}\left(P / P^{2}\right)=$ alt $(\mathscr{L})$.

THEOREM 4.1. If $\mathscr{L}$ is a regular local lattice, then 0 is prime.

Proof. If alt $(\mathscr{L})=0$, then the result is clear. The proof will proceed by induction. Assume alt $\mathscr{L}=d \geqq 1$. Let $P_{0}<P_{1}<\cdots<P_{d}=P$ be a prime chain in $\mathscr{L}$ and let $A_{1}, \ldots, A_{d}$ be a principal element of $\mathscr{L}$ with $P=A_{1} \vee \cdots \vee A_{d}$. Then $\mathscr{L} \mid P_{0}$ is again regular with maximal element $P=P / P_{0}=A_{1} / P_{0} \vee \cdots \vee A_{d} / P_{0}$. Since $P / P_{0}$ is not the join of fewer than $d$-principal elements [1], it is clear that $A_{i} / P_{0} \neq 0 / P_{0}$ for $i=1, \ldots, d$. Then $\left(\mathscr{L} \mid P_{0}\right)\left|\left(A_{1} / P_{0}\right)=\mathscr{L}\right|\left(A_{1} \vee P_{0}\right)$ has altitude $d-1$ (Lemma 1.8) and is regular since $P /\left(A_{1} \vee P_{0}\right)=A_{2} /\left(A_{1} \vee P_{0}\right) \vee \cdots \vee A_{d} /\left(A_{1} \vee P_{0}\right)$. Hence by the inductive hypothesis, $A_{1} \vee P_{0} \doteq 0 /\left(A_{1} \vee P_{0}\right)$ is prime in $\mathscr{L} /\left(A_{1} \vee P_{0}\right)$, and therefore also in $\mathscr{L}$.

Now, $P=P / A_{1}$ is the join of the $d-1$ principal elements $A_{2} / A_{1}, \ldots, A_{d} / A_{1}$ in $\mathscr{L} \mid A_{1}$, so $\mathscr{L} \mid A_{1}$ has altitude $\leqq d-1$ [1] and therefore $=d-1$, (Lemma 1.8). Hence, by the inductive hypothesis, $A_{1}=0 / A_{1}$ is prime in $\mathscr{L} / A_{1}$, and therefore also in $\mathscr{L}$. Since $A_{1} \leqq A_{1} \vee P_{0}$, and since $\mathscr{L} \mid A_{1}$ and $\mathscr{L} /\left(A_{1} \vee P_{0}\right)$ both have altitude $d-1$, so that $A_{1}$ and $A_{1} \vee P_{0}$ have the same depth in $\mathscr{L}$, it is immediate that $A_{1}=A_{1} \vee P_{0}$, whence $P_{0} \leqq A_{1}$. Then $P_{0}=P_{0} \wedge A_{1}=\left(P_{0}: A_{1}\right) A_{i}=P_{0} A_{1}$ (since $P_{0}$ is prime $A_{1} \$ P_{0}$ ), so $P_{0}=0$ by Lemma 1.3. Q.E.D.

COROllary 4.2. Let $\mathscr{L}$ be a regular local lattice of altitude $d$, and let $A_{1}, \ldots, A_{d}$ be principal elements with $P=A_{1} \vee \cdots \vee A_{d}$. Then $A_{1} \vee \cdots \vee A_{i}$ is prime for $i=0, \ldots, d$.

Proof. By Lemma 1.8, it follows (by induction on $i$ ) that $\mathscr{L} /\left(A_{1} \vee \cdots \vee A_{i}\right)$ is regular of altitude $d-i$, so that $0 /\left(A_{1} \vee \cdots \vee A_{i}\right)=A_{1} \vee \cdots \vee A_{i}$ is prime in $\mathscr{L} /\left(A_{1} \vee \cdots \vee A_{i}\right)$ and therefore also in $\mathscr{L}$. Q.E.D.

COROllary 4.3. Let $A$ be a principal element of the regular local lattice $\mathscr{L}$, with $A \leqq P$ and $A \ddagger P^{2}$. Then $A$ is prime in $\mathscr{L}$. 
Proof. Let $\mathscr{L}$ be a regular of altitude $d$, so that $\operatorname{dim}\left(P / P^{2}\right)=d$, (Theorem 1.4). Now, $\left(A \vee P^{2}\right) / P^{2} \cong A /\left(P^{2} \wedge A\right)=A /\left(P^{2}: A\right) A \cong I /\left(P^{2}: A\right)\left(\right.$ Lemma 1.1) so $\left(A \vee P^{2}\right) / P^{2}$ has dimension 1 and $P /\left(A \vee P^{2}\right)$ has dimension $d-1$. Let $A \vee P^{2}=C_{1}<C_{2}<\cdots$ $<C_{d}=P$ be a composition chain for $P /\left(A \vee P^{2}\right)$. For each $i=2, \ldots, d$ let $A_{i}$ be a principal element of $\mathscr{L}$ such that $A_{i}<C_{i}$ and $A_{i} \pm C_{i-1}$. Then $C_{i-1} \vee A_{i}=C_{i}$, for $i=2, \ldots, d$. Set $A=A_{1}$. Then $P=C_{1} \vee A_{2} \vee \cdots \vee A_{d}=P^{2} \vee A_{1} \vee \cdots \vee A_{d}$, so $P=A_{1} \vee \cdots \vee A_{d}$ by Lemma 1.3 , so $A_{1}$ is prime by Corollary 4.2. Q.E.D.

Corollary 4.4. Let $A, B$ be elements of a regular local lattice $\mathscr{L}$, with $A \leqq P^{n}$, $A \nsubseteq P^{n+1}$ and $B \leqq P^{m}, B \nsubseteq P^{m+1}$. Then $A B \leqq P^{n+m}$ and $A B \leqq P^{n+m+1}$.

Proof. Let $\mathscr{L}$ be regular of altitude $d$, with $P=A_{1} \vee \cdots \vee A_{d}\left(A_{i}\right.$ principal). Then the maximal element of $\mathscr{R}(\mathscr{L}, P)$ is the join of the $d+1$ principal elements $I^{(-1)}$, $A_{1}^{(1)}, \ldots, A_{d}^{(1)}$ (Lemma 2.3 and Theorem 2.7$)$, so $\mathscr{R}(\mathscr{L}, P)$ is a regular local lattice of altitude $d+1$ (Theorem 2.17). Hence $I^{(-1)}$ is prime (Corollary 4.2). Now, assume $A, B \in \mathscr{L}, A \leqq P^{n}, A \leqq P^{n+1}$, and $B \leqq P^{m}, B \leqq P^{m+1}$. Then $I^{(-1)}$ has $i$ th coordinate $P^{i+1}$, so $A^{(n)} I^{(-1)}$ and $B^{(m)} \npreceq I^{(-1)}$. Hence $(A B)^{(n+m)}=A^{(n)} B^{(m)} \leq I^{(-1)}$, and therefore $A B \nsubseteq P^{n+m+1}$. Q.E.D.

Hence the degree function on a regular local lattice is a valuation.

It is noted that, as for local rings, if $\mathscr{L}$ is regular of altitude 1 , then Theorem 2.7 can be strengthened to say that every element $A \neq 0$ is a power $P^{n}$ of the maximal element $\left(I=P^{0}\right)$. For in this case, $P$ is principal, so if $n$ is the largest $i$ such that $A \leqq P^{i}$, then $A=A \wedge P^{n}=\left(A: P^{n}\right) P^{n}$, so that if $P^{n} \neq A$, then $A \leqq P P^{n}=P^{n+1}$. Hence if $\mathscr{L}$ is a regular local lattice of altitude 1 , then $\mathscr{L}$ is isomorphic to the lattice of ideals of a regular local ring, and $\mathscr{L}^{*}=\mathscr{L}-\{0\}$ is a Gaussian semigroup. This second property also holds in the semigroup of principal elements of a regular local lattice of altitude 2 , as is easily seen.

\section{REFERENCES}

1. R. P. Dilworth, Abstract commutative ideal theory, Pacific J. Math. 12 (1962), 481-498.

2. M. Nagata, Local rings, Interscience, New York, 1962.

3. D. G. Northcott, Ideal theory, Cambridge Univ. Press, New York, 1963.

4. D. Rees, A-transforms of local rings and a theorem on multiplicities of ideals, Proc. Cambridge Philos. Soc. 57 (1961), 8-17.

5. O. Zariski and P. Samuel, Commutative algebra, Vol. II, Van Nostrand, Princeton, N. J., 1960.

UNIVERSITY OF IOWA, IOWA City, IOWA 